Aus der Königl. Universitäts-Frauenklinik zu Berlin. Direktor: Geheimrat Prof. Dr. E. Bumm.

\title{
Anatomische Befunde bei mit Mesothorium und Röntgenstrahlen behandelten Carcinomen.
}

\author{
Von \\ Dr. Paul Haendly, \\ Assistent. \\ (Hierzu Tafel IV-V.)
}

In der Berliner Medizinischen Gesellschaft und auf dem diesjährigen Kongress der Deutschen Gesellschaft für Gynäkologie in Halle hat Bumm (1) über die klinischen Erfolge bei den von uns mit strahlender Energie behandelten Carcinomen berichtet, nachdem wir schon im vergangenen Jahre $(1,5)$ über einzelne Fälle Mitteilung gemacht hatten. Wir baben die behandelten Fälle an fortlaufenden Probeexzisionen mit freundlicher Unterstützung von Rob. Meyer in bezug auf die auftretenden Veränderungen untersucht. Die Erfahrungen, die wir dabei sammelten, deckten sich, was die Beeinflussung der Carcinomzellen anbetrifft, mit denen anderer Autoren. Wir sahen entsprechend der Dauer der Behandlung eine Abnahme des Carcinoms, ein Zugrundegehen der Carcinomzellen bis zu einem vollständigen Schwund an den der Probeexzision zugänglichen Stellen. Daneben fanden wir weitgehende Veränderungen des Bindegewebes im Sinne einer Sklerose und hyalinen Degeneration der Fibrillen, Intimaveränderungen und Sklerose der Adventitia an den Gefässen. Das nach dem Untergange der Carcinomzellen restierende Gewebe bestand, abgesehen von dem hyalin degenerierten Bindegewebe, in einzelnen Fällen aus einem schwach gefärbten Granulationsgewebe. Es ergab sich also aus diesen Untersuchungen einwandfrei, dass Carcinomgewebe durch die Behandlung mit Mesothorium- und Röntgenstrahlen zum Schwinden gebracht werden kann. Ueber den Ersatz des zugrunde gegangenen Gewebes aber war kein genügendes Urteil zu gewinnen, 
ebensowenig wie über die Frage der Tiefenwirkung der Strahlen. Gerade die Tiefenwirkung der Strahlen aber ist zur Beurteilung der Heilbarkeit des Carcinoms von der grössten Wichtigkeit. Um in dieser Frage zu einem Ergebnis zu kommen, genügt die Untersuchung von Probeexzisionen, die aus den vor der Behandlung carcinomatösen Partien gemacht werden, nicht; denn es ist in dem narbig geschrumpften und zusammengezogenen Gewebe nicht möglich, genügend ausgiebige, alle Teile treffende.Exzisionen zu machen. Diese Frage lässt sich vielmehr nur an Organen entscheiden, die nach voraufgegangener Strahlenbehandlung in toto der Untersuchung zugänglich gemacht werden. Wir sind im Besitze von 3 operativ und 2 bei der Sektion gewonnenen Präparaten, die ein Bild von der bis jetzt erreichten Tiefenwirkung geben können. Die Fälle sind ausserdem auch noch vor der Aufnahme der Behandlung histologisch, zum Teil wiederholt untersucht worden. Des weiteren können noch die von Aschoff, Krönig und Gauss (2) mitgeteilten Sektionsprotokolle, ferner der von Döderlein (3) operativ gewonnene Uterus und endlich ein von Wickham (4) veröffentlichter Fall zur vorläufigen Entscheidung und Beurteilung des Erreichten herangezogen werden.

In den von Aschoff, Krönig, Gauss (2) veröffentlichten Fällen handelt es sich um je ein Magen-, Cervix- und Mammacarcinom. Ueber die Art der Behandlung und die verabreichte Strahlenmenge ist nichts gesagt. Bei dem ersten Fall fand sich bei der Sektion mikroskopisch im Magen nichts mehr von den "alten carcinomatösen Wucherungen". Dagegen zeigten sich in einigen spärlichen Schleimresten im Geschwürsgrund und in der Mnskulatur noch nvereinzelte grosskuglige, mit grösseren Kernen versehene, als Ca.-Zellen anzusprechende Gebilde". "Von irgendwelchen frischen Krebszellwacherungen war nichts mehr zu entdecken." In einer weiteren Exzision aus dem Ulcus ist die verdickte Subserosa und Muscularis frei von Carcinom und nur im Geschwürsgrund in dem dort befindlichen Granulationsgewebe lagen „zerstreute kleine Gruppen von polymorphen, sich gegenseitig abplattenden, zum Teil sich schalenförmig umgebenden, mehr wie Pflasterepithel aussehenden Krebszellen, deren Protoplasma feinkörnige Verfettung aufweist". In den portalen Lymphknoten, in der Leber und in den beiden Ovarien fanden sich Carcinommetastasen.

Kurz zusammengefasst kann also von diesem Fall gesagt werden: lebensfrische Carcinomzellen fanden sich im Primärtumor 
nicht, doch waren noch Reste veränderter und morphologisch geschädigter Carcinomzellen vorhanden. Die Metastasen waren unbeeinfiusst.

Im zweiten Fall, einem inoperablen Cervixcarcinom, hatten die Probeexzisionen vor dem Exitus dauernd Carcinom ergeben, das aber einige Veränderungen seines Charakters darbot. Mikroskopisch fand sich nun an dem Leichenpräparat eine nausgedehnte oberflächliche Nekrose", darunter eine "ausgebreitete carcinomatöse Wucherung". Auffällig war an dem Carcinom der verschiedene Charakter der Neubildung: in der Tiefe Krebsstränge sehr zahlreich und aus Zellen bestehend, die den basalen Elementen gleichen, gegen die Oberfläche zu grosse „riesige Zellen mit entsprechend grossen Riesenkernen"; die Kerne sind hier unregelmässig geformt, sehr chromatinreich und zeigen Vakuolenbildung. In beiden Schichten finden sich Perlbildungen. Der Uebergang zwischen den beiden Schichten ist ein ziemlich plötzlicher.

Zusammenfassend kann za diesem Fall bemerkt werden, dass eine geringfügige Beeinflussung des Carcinoms nur in den oberflächlichen Schichten stattgefunden hat.

In dem dritten Fall endlich lag ein doppelseitiges Mammacarcinom vor. Bei der Sektion fand sich an der linken Mamma in einem "mehr der Oberfl̈̈che entsprechenden Gebiet" noch Krebsgewebe und zwar machten die Zellen den Eindruck "wohlerhaltener Lebensfähigkeit". Auch hier liessen sich Lmänderungen des Zellcharakters feststellen insofern, als am Rande des Knotens "die relativ kleinen Krebszellen der zentralen Gebiete in grössere, zum Teil auffallend grosse, mehr plattenepithelähnliche Elemente übergehen". Ein sehr bemerkenswerter Befund konnte an dem Mammadrüsengewebe erhoben werden. Neben einer starken Bindegewebswucherung um die Drüsenkanälchen fand sich eine "Metaplasie" des Drüsenepithels in Plattenepithel unter gleichzeitiger Wucherung der Zellen, so dass die Lumina der Kanälchen verschlossen waren. Ob dieser "Metaplasie" eire stärkere Schädigung mit Epithelverlust vorausgegangen war, konnte Aschoff nicht entscheiden.

Auf der rechten Seite fand sich unter der „oberflächlichen nekrotischen Schicht ein spindelzellenreiches von atrophischen pigmentierten Muskelresten durchsetztes Gewebe", in dem keine Carcinomreste gefunden wurden. „In den axillaren Lymphknoten lässt sich ausgedehnte Krebswucherung feststellen." 
Es kann also über diesen letzten Fall zusammenfassend festgestellt werden, dass sich in der einen Mamma neben weniger gut erhaltenen Carcinomzellen mit teilweiser Charakteränderung der Zellen eine Metaplasie des Drüsenepithels und eine Bindegewebswucherung, in der anderen Mamma neben Veränderungen des Bindegewebes und Untergang der Muskulatur kein Carcinom fand. Eine Beeinflussung der Drüsenmetastasen war nicht festzustellen.

In dem Döderlein'schen Falle (3) handelte es sich um ein inoperables Carcinom der Portio, das sich bis in das Corpus hinauf erstreckte. Eine Infiltration des rechten Parametriums bis an die Beckenwand fixierte den Uterus fest. Die Patientin wurde mit Röntgenstrahlen und Mesothorium behandelt und zwar mit dem Erfolg, dass das Carcinom nach zirka 3 Monaten operabel erschien. In den Probeexzisionen aus der Portio konnte kein Carcinom mehr nachgewiesen werden. Bei der Operation stellte sich aber heraus, dass eine radikale Entfernung des Tumors nicht möglich war. Es musste der Ureter unterbunden und reseziert und ein am rechten "Beckenknochen breitbasig fixierter Rest" zurückgelassen werden. Die mikroskopische Untersuchung ergab "an der Portio und an der Cervix keine carcinomatösen Herde mehr". In dem Parametrium aber fand sich noch unverändertes Carcinomgewebe.

Dieser Fall zeigte also ein Schwinden des Carcinoms in der zuerst ergriffenen Portio und Cervix, ein Schrumpfen der parametranen Infiltration bis auf einen durch die Strahlenbehandlung unbeeinflussten Rest von Carcinom, der am Becken so fest fixiert war, dass er nicht völlig entfernt werden konnte.

Der von Wickham (4) beobachtete Fall, der später von Tuffier operiert worden ist, betraf eine 56jährige Frau, die an einem "Epitheliom des Uterus" litt. Der Tumor, der die vordere und hintere Lippe des Uterus mit blumenkohlartigen Wucherungen überzog, war inoperabel. Nach zirka 4 monatiger Behandlung wurde der Fall für operabel erklärt und die Hysterektomie vorgenommen. Das Carcinom war von den Muttermundslippen "vollständig beseitigt, wie weggekratzt", nur auf der vorderen Lippe bestand noch ein ,linsengrosses Knötchen". Die von Mauté vorgenommene mikroskopische Untersuchung ergab "Carcinom des Uterushalses ohne Beteiligung des gesamten Uterus".

Wie weit eine Schädigung des Carcinoms zu konstatieren 
war, wie tief sie ging, ist leider nicht bemerkt. Wir können daher als einziges Zeichen des Erfolges die Tatsache vermerken, dass ein für inoperabel erklärter Tumor durch die Behandlung mit Radium operabel wurde.

Auf die Schlussfolgerungen aus diesen Fällen werde ich weiter unten eingehen, nachdem ich zunächst über unsere 5 Fälle berichtet habe.

Von dem ersten der von uns beobachteten Fälle, der im Juni 1912 in der hiesigen Gesellschaft für Geburtshilfe und Gynäkologie (5) noch vor der Operation vorgestellt und von Bumm (1) und Robert Meyer (6) schon mitgeteilt worden ist, soll nur kurz das Wesentliche hervorgehoben werden. Die 45 jährige Patientin wurde von uns Ende Mai wegen eines inoperablen Cervixcarcinoms, das nach links auf die Vagina und das Parametrium übergegriffen hatte, aufgenommen und mit Röntgenstrahlen behandelt. Sie erhielt 1600 x. Im Oktober hatte sich der Befund derartig verändert, dass ar eine radikale Entfernung des Carcinoms gedacht werden konnte. Aus dem grossen Carcinomkrater war eine enge Höhle geworden, die den Finger kaum eindringen liess, und aus der die Curette nur noch dünne Gewebsstücke entfernen konnte. Die Infiltration im linken Parametrium war geschrumpft und gegen die Umgebung scharf abgesetzt. Die Operation konnte radikal vorgenommen, und die Patientin nach normaler Rekonvaleszenz entlassen werden. Aus dem mikroskopischen Befund (T. 214), über den Robert Meyer (6) schon berichtet hat, wäre noch folgendes hervorzuheben: Die Cervix ist kontinuierlich mit Carcinom bis herauf über den inneren Muttermund bekleidet, das die grossalveoläre Form des Plattenepithelcarcinoms zeigt. Nur eine kleine Partie macht den Eindruck eines Skirrhus. Die Carcinomzellen sind überall gut erhalten und zeigen zahlreiche Mitosen. Die nächste Umgebung der Carcinomzellen besteht aus einem stark lymphozytär infiltrierten Bindegewebe, in dem sich kaum einzelne Bindegewebszellen und nur wenige noch gut erhaltene Muskelzellen finden, dagegen ein Netz teils zarter, teils grober Fibrillen. "Das ist in der Tat das Auffallendste des Falles, dass im übrigen die Wand der Cervix ausserordentlich stark sklerosiert ist. Nur wenige Bindegewebskerne liegen in einem starren Fasergewebe, dessen Fibrillen vielfach zu derben Strängen zusammengeschweisst sind." Aber auch in der sklerosierten Wand finden sich noch einzelne Carcinom- 
54 Haendly, Mit Mesothorium und Röntgenstrahlen behandelte Carcinome.

stränge. Das Parametrium ist in auffällig starkem Grade von grossen zirkumskripten Infiltrationen (Lymphozyten) durchsetzt. Zwei derb geschwollene Lymphknoten sind frei von Carcinom.

Das Besondere an diesem Falle war, dass ein inoperables Cervixcarcinom während der fünfmonatigen Beobachtung und Behandlung mit Röntgenstrahlen nicht weiter fortgeschritten war, sondern im Gegenteil der Operation soweit zugänglich gemacht werden konnte, dass eine Radikalentfernung möglich war. Die mikroskopische Untersuchung enttäuschte allerdings insofern, als sie in einem gewissen Gegensatze zu der auffallenden klinischen Besserung stand. Das Auffallendste bei der mikroskopischen Untersuchung war die starke Sklerose der Bindegewebsfibrillen, während die Carcinomzellen lebensfrisch waren, soweit das aus der guten Färbefähigkeit und dem unveränderten Aussehen geschlossen werden konnte. Ein Uebergreifen auf die Lymphdrüsen hatte in dieser Zeit der konservativen Behandlung nicht stattgefunden.

In dem zweiten unserer Fälle handelte es sich um eine 35 jährige Frau H. W., die mit Mesothorium bestrahlt wurde.

Der mikroskopische Befund (Nr. 3151) bei der Aufnahme war folgender: Alveoläres Carcinom mit mässig viel Kernteilungsfiguren. Starke kleinzellige Infiltration des Bindegewebes, die hauptsächlich aus Lymphozyten besteht. Stellenweise findet sich die kleinzellige Infiltration auch zwischen den Carcinomzellen. Viele Gefässe, bei denen es hier und da zur Stase gekommen ist. Einzelne Hämorrhagien. Adventitia der Gefässe stellenweise sklerotisch, desgleichen vereinzelt eine Sklerose der Bindegewebsfibrillen. Viel normale Cervicaldrüsen.

Die Patientin erhielt innerhalb von etwa 14 Tagen 9350 mgr.Stunden mit 0,1-1,0 mm Silber-Blei-Filter per vaginam appliziert. Die Operation bot bis auf die Ablösung der Rektums keine besonderen Schwierigkeiten. Die präparatorische Auskratzung und das Ausbrennen des Carcinomtrichters vor der Operation konnte unterbleiben, da „aus der geschwürig zerfallenden Carcinomhöhle unter der Mesothoriumbehandlung eine nach Innen zu trichterförmig verlaufende glattwandige, ein geruchloses eitrig-schleimiges Sekret absondernde Höhle entstanden war". Auf der rechten Seite wurde aus dem Gefässdreieck ein Paket Drüsen entfernt.

Makroskopisch präsentierte sich das Präparat (T.461, Fig. 1) folgendermassen: Corpus uteri von annähernd normaler Grösse. Die Schleimhaut glasig, nach dem Orificium internum zu leicht blutig 
imbibiert, hypertrophisch. Cervix verdickt von derber Konsistenz. Die Oberfläche des Cervicalkanals uneben, graurot. Der ganze Cervicalkanal ist ringsum von einer derben, markigen, knotigen Masse umgeben, die den grössten Teil der Wand einnimmt, seitlich in die Parametrien, dorsal fast bis an die Serosa heranreicht und nur vorn etwa $1 \mathrm{~cm}$ von der Oberfläche entfernt bleibt. Von der Portio nimmt das Carcinom die hintere Lippe vollständig ein, dagegen vorn nur die seitlichen und inneren Schichten, während die äusseren und mittleren frei sind. Die untere Fläche der Portio und die mitexstirpierten Scheidengewölbe sind braun verfärbt (Arg. nitr.?). Adnexe ohne Besonderheiten.

Die mikroskopische Untersuchung ergab folgendes (T. 461): Die Oberfläche der Scheide ist in den oberen Lagen sehr kernarm und zeigt eine braune Farbe. Die Portio wird ebenfalls von einem schmalen braunen Streifen überzogen, der Reste einer kleinzelligen Infiltration erkennen lässt. Unter dieser Schicht findet man eine breite Zone schwachgefärbter, lockerer Granulationen. Sehr reichlich finden sich in dieser Zone Gefässe, die aber alle Veränderungen ihrer Wand im Sinne einer hyalinen Degeneration der Adventitia zeigen. Endlich finden sich hier einige Carcinomnester, deren Zellen alle Stadien des Unterganges zeigen: die Zellen sind in einandergeflossen und erinnern an Syncytium, das Protoplasma ist ungleichmässig gefärbt und weist grosse Vakuolen auf, die Kerne zeigen eine unregelmässige, zum Teil halbmondförmige Gestalt und sind ebenfalls schlecht und unregelmässig gefärbt, zum Teil enthalten sie nur noch einige dunkel gefärbte Klümpchen, während der übrige Teil des Kerns überhaupt nicht mehr tingiert ist. Die Zellen liegen isoliert oder zu mehreren in kleinen Hohlräumen: Ungefähr in der Höhe des Os ext. finden sich einige Cervicaldrüsen, von denen die einen mit normalem Zylinderepithel bekleidet sind, während in den andern das Epithel teilweise durch Carcinomzellen abgehoben erscheint. In nächster Nähe dieser Cervixdrüsen, dicht unter der oberflächlichen nekrotischen Schicht, finden sich zwei grössere Carcinomzellennester, deren Zellen verschiedene Grade des Unterganges zeigen: das Protoplasma ist verschieden stark gefärbt, gekörnt und weist grosse Vakuolen auf, so dass die Kerne hier und da wie in einem Hohlraum liegen, der von einem feinen gefärbten Ring umgeben ist. Auch die Kerne weisen Zeichen des Unterganges auf: neben wenigen gut gefärbten findet man viel hyperchromatische, andere Kerne zeigen nur noch einige meist 
exzentrisch gelagerte, dunkel tingierte Klümpchen, während der übrige Teil des Kerns nicht mehr gefärbt ist. Wieder andere Kerne sehen wie angefressen aus oder sind vollständig im Zerfall, endlich findet man in manchen Zellen nur noch einige unregelmässig gelagerte dunkel gefärbte Körnchen - Pyknose - oder überhaupt nichts mehr - der Kern ist völlig zugrunde gegangen - Karyolyse. Die Wand ist entsprechend der makroskopisch erkennbaren Ausdehnung mikroskopisch mit Carcinommassen durchsetzt, die in ausgedehntem Maasse im Zerfall begriffen sind (Fig.2). Dieser Untergang der Zellen findet sich vor allem und scheint zu beginnen im Zentrum der Krebsalveolen, während sich am Rande der Alveolen, die Detritusmassen umgebend, noch mehr oder weniger gut erhaltene Carcinomzellen zeigen. Doch sind auch sehr reichlich Hohlräume, die den Carcinomalveolen entsprechen, einzig und allein angefüllt mit Detritus. Dieser Zerfall von Carcinom findet sich sowohl in den der Oberfläche zu liegenden Schichten, wie in der Tiefe. Daneben liegen aber auch Carcinomzellen meist in kleinen Nestern und Strängen zusammen, die tinktoriell und ihrer Form nach absolut den Eindruck der Lebensfähigkeit machen. Die gut erhaltenen Zellen (Fig. 3) finden sich besonders an der Peripherie der Neubildung und in der Tiefe, doch ziehen auch an verschiedenen Stellen Züge lebensfrischer Carcinomzellen zwischen zugrunde gehenden Zellbaufen bis dicht unter die nekrotische Oberfläche. Mehrfach ist Neigung zur Verhornung zu konstatieren. Endlich zeigt sich an verschiedenen Stellen eine starke Verfettung der Carcinomzellen. Keine Kernteilungsfiguren. Das Bindegewebe der Septen zeigt eine wechselnd starke Sklerose und hyaline Degeneration der Fibrillen. Vielfach findet sich hier auch eine kleinzellige Infiltration. Die Muskulatur der Wand ist im allgemeinen gut erhalten, das Bindegewebe zeigt stellenweise eine Sklerose und hyaline Degeneration: Die Gefässe weisen hier und da eine Verdickung der Intima und eine Sklerose der Adventitia auf.

In einigen der exstirpierten Lymphdrüsen sind Carcinomherde vorhanden, die einen zentralen Zerfall zeigen. Die Zellen in der Peripherie sind aber völlig lebensfrisch.

Zusammenfassend ist über diesen zweiten Fall zu sagen, dass bei dem sehr weit vorgeschrittenen Carcinom trotz der kurzen Behandlung ein Einfluss der Mesothoriumstrahlen unverkennbar ist: in ausgedehntem Masse findet sich ein Zugrundegehen der Carcinomzellen, doch sind daneben in der Tiefe und besonders in der Umgebung der Neubildung noch reichlich gut erhaltene Carcinom- 
stränge vorhanden, die hier und da bis unter die Oberfläche hin sich erstrecken. Die Bindegewebsfibrillen zeigen eine Sklerose und hyaline Degeneration, die Gefässe stellenweise eine starke Verdickung der Intima und eine Sklerose der Adventitia. Ein Einfluss auf die Metastasen in den Lymphdrüsen (Fig. 4) ist nicht vorhanden, denn einen zentralen Zerfall trifft man auch sonst an.

Der dritte Fall betraf eine 57jährige Frau M. F. mit einem Cervixcarcinom. Der mikroskopische Befund bei der Aufnahme war folgender:

Nr. 3135. Alveoläres PJattenepithelcarcinom. Zwischen breiten Zügen von stark infiltriertem Bindegewebe finden sich kleinere und grössere Haufen und Stränge von gut tingierten im allgemeinen kleinen Carcinomzellen.

Die vor der Operation 3 Wochen lang mit Mesothorium bestrahlte Patientin erhielt 13320 mgr-Stunden mit der gleichen Filterung wie der vorhergehende Fall appliziert. Aus der kraterförmigen Höhle wurde ebenfalls ein glattwandiger zusammengezogener Trichter, der ein Auslöffeln und Ausbrennen vor der Operation erübrigte. Die Operation verlief absolut typisch und bot keinerlei Schwierigkeiten. Aus dem rechten Gefässdreiek wurden 2 kleine Drüsen entfernt. Ueber das Präparat (Fig. 5) ist makroskopisch zu bemerken (T. 462): kleiner Uterus, Corpus ziemlich kurz, in der Hinterwand ein pflaumengrosses Myom, submukös vorspringend, daneben noch mehrere kleinere Knoten. Die Cervix ist lang, auf dem Durchschnitt derb-fibrös. Die untere Hälfte des Cervicalkanals zeigt eine glatte braun verfärbte Oberfläche, im oberen Teil ist sie rauh, höckerig, von grauroter Farbe. Im Bereich dieser flachen. Wucherungen ist die Wand an einzelnen Stellen von sehr dünnen, gelbgrauen, markigen Strängen durchzogen. In der Medianlinie unterhalb der Douglastasche fällt eine flache Verdickung der Uteruswand auf. Adnexe sind ohne Besonderheiten, senil geschrumpft.

Mikroskopisch (T. 462) fand sich folgendes: die Portio und etwa die untere Hälfte des Cervicalkanals wird überzogen von einer Zone, in der das Gewebe wohl durch das zur Desinfektion verwandte Arg. nitrieum braun gefärbt ist und in der die einzelnen Zellen gut erhalten sind. Dieser Zone haften an einigen Stellen mehr oder weniger dicke Stückchen nekrotischen Gewebes an, in dem keine Kerne mehr tinktoriell darstellbar sind. Das Gewebe unter dieser Zone ist stellenweise stark kleinzellig infiltriert — viel 
polynukleäre Leukozyten, Fremdkörperriesenzellen. Das Bindegewebe zeigt eine Sklerose und hyaline Degeneration der Fibrillen (Fig.9) und stellenweise eine starke Verarmung an Bindegewebskernen. Es finden sich hier zirkumskripte Anhäufungen von kleinzelliger Infiltration, die bald mehr aus polynukleären Leukozyten, bald mehr aus Lymphozyten bestehen. Die Oberfiäche oberhalb der Mitte der Cervix zeigt eine mehr oder weniger breite Zone von Granulationsgewebe, dem hier und da Stücke nekrotischen Gewebes aufliegen. An einzelnen Stellen findet sich in diesem Granulationsgewebe eine ausgedehnte Neubildung von Bindegewebe (Fig. 8). Während sich in der unteren Hälfte der Cervix keine Carcinomzellen oder nur Reste solcher finden, liegen oberhalb der Mitte der Cervix grössere und kleinere Nester von Carcinomzellen - im ganzen aber an Zahl spärlich — fast über die ganze Dicke der Wand verteilt. Diese Nester nehmen von der Oberfläche der Cervixhöhle nach der Serosa zu an Grösse $a b$ und bestehen teilweise nur aus 2-3 Zellen; stellenweise findet man nur isoliert liegende Riesenzellen, ein- oder mehr-, bis vier- und sechskernig. Vielfach liegen diese Zellen in kleinen Hohlräumen inmitten des Bindegewebes. An den Carcinomzellen fällt zunächst einmal in die Augen, dass das Protoplasma auffallend hell gefärbt ist, während die Kerne verschieden stark tingiert sind. Fast durchgehends zeigen die Zellen mehr oder weniger starke Zeichen des Unterganges. An der Oberfläche, nach der Cervixhöhle zu, sind die Carcinomnester an verschiedenen Stellen in die allgemeine oben erwähnte Gewebsnekrose einbezogen. Die Zellen sind als selbständige Gebilde nicht mehr zu erkennen, sie sind vielmehr miteinander verschmolzen und haben einen syncytialen Charakter (Fig. 7) angenommen. Das Protoplasma bildet eine homogene, zuweilen schollige, mit Hämatoxylin (Weigert)-van Gieson verschieden dunkelbraun gefärbte Masse, in der die Kerne unregelmässig verteilt sind. Die Kerne sind zum Teil aufgequollen, bläschenförmig bald hell, bald dunkel gefärbt, zeigen dunkel tingierte Körnchen oder ein dunkel tingiertes Netzwerk mit Vakuolen dazwischen, zum Teil zeigen die Kerne mehr eine Spindel- oder Stäbchenform und sind sehr dunkel tingiert, so dass Details nicht erkennbar sind (Fig. 6, 9). An den tiefer in der Wand liegenden Carcinomnestern, deren letzteEtappen fast dicht unter der Serosaliegen, finden sich neben ganz wenigen Nestern mit relativ gut erhaltenen Zellen alle Stadien des Unterganges. Auch hier sind die Zellen vielfach syncytial verschmolzen. Das Protoplasma ist homogen, 
bald ganz blass, bald dunkler gefärbt. Die Kerne zeigen nur an einer Stelle ein annähernd normales Aussehen, an anderen Stellen, und diese sind in der Mehrzahl, sind die Kerne gequollen, oder zeigen Stäbchenform. Tinktoriell sind sie vielfach hypo- oder hyperchromatisch. In vielen Kernen finden sich Vakuolen, oft so stark hervortretend, dass man von der Kernsubstanz nur noch ein feines Netzwerk vor sich hat. Viele Carcinomnester liegen in Hohlräumen, die anscheinend entstanden sind durch Untergang von Carcinomzellen, denn man findet alle Uebergänge von geschlossen liegenden Carcinomzellnestern und Hohlräumen, oft spaltförmigen, in denen nur noch einzelne Protoplasmaschollen liegen, umgeben von Leukozyten und Rundzellen. Ueberall dort, wo ein Untergang: von Carcinomzellen zu beobachten ist und wo sich dadurch entstandene Hohlräume finden, sind zahlreiche Leukozyten und Rundzellen, oft in breiten Zügen und Haufen, anzutreffen, die die Zellreste umlagern und in sie eindringen (Fig. 7). Endlich findet man diffus über die Wand zerstreut isolierte Carcinomzellen, die fast alle einen gequollenen, mehr oder weniger gut gefärbten Kern zeigen und ein meist blass tingiertes, homogenes Protoplasma. Vielfach ist von dem Protoplasma nichts mehr zu sehen, der Kern, hypochromatisch nur noch als Schatten erkennbar. Zum Schluss sei noch erwähnt, dass sich eine mässig ausgedehnte Verhornung findet (Fig. 7).

Die Lymphdrüsen sind frei von Carcinom.

Ueberblicken wir die in diesem Falle erhobenen Befunde, so können wir sagen, dass es unter Einwirkung der Strahlen zu einer Veränderung der Carcinomzellen gekommen ist, die auf eine erhebliche Schädigung ihrer Lebensfähigkeit schliessen lässt. Gut erhaltene Carcinomzellen finden sich überhaupt kaum mehr. Im Bindegewebe ist es neben einer Sklerosierung der Fibrillen auch $\mathrm{zu}$ einer ausgedehnten Bindegewebsneubildung gekommen.

Die bisher beschriebenen Fälle betrafen Tumoren, die operativ gewonnen waren. In den noch folgenden beiden Fällen handelt es sich um Sektionspräparate. Die Frauen waren mit inoperablen Carcinomen in die Klinik eingetreten und nach lange fortgesetzter Behandlung an den Folgen ihres Leidens trotz der Behandlung gestorben.

Bei der ersten dieser Patientinnen, Frau Pr., 76 Jahre alt, lag ein ausgedehntes Cervixcarcinom vor, das bei dem Alter und dem sehr schlechten körperlichen Zustand der Patientin als 
inoperabel bezeichnet werden musste, zumal das Carcinom auf die rechte Seite der Scheide und nach vorn zu übergegriffen hatte. Die Patientin - aufgenommen am 23. IX. 12 - wurde zuerst mit Röntgenstrahlen allein, später mit Mesothorium behandelt und erhielt $1940 \mathrm{H}=3880 \mathrm{x}$ und $29075 \mathrm{mgr}-\mathrm{Std}$. Mesothorium mit einem dünnen Silberfilter per vaginam appliziert. Zunächst besserte sich das klinische Bild in der bekannten Weise: die Blutung und Jauchung liess nach, das Carcinom schrumpfte, die ganze Höhle verkleinerte sich sehr stark. In der Tiefe aber wucherte das Carcinom weiter und wuchs, wie eine Blasenuntersuchung am 28. I. 13 zeigte, speziell nach der Blase zu, deren Schleimhaut durch die Knoten vorgewölbt wurde. Anfangs März begann eine fortschreitende Nekrose, die zunächst den carcinomatös infiltrierten Blasenrand ergriff, dann immer weiter um sich greifend, die ganze Basis der Blase und das Scheidengewölbe zerstörte. Patientin fieberte und am 7. V. erfolgte der Exitus.

Die während der Behandlung vorgenommenen mikroskopischen Untersuchungen an Probeexzisionen hatten folgendes Ergebnis:

23. X. 12. Nr. 2870. Alveoläres Carcinom, an einzelnen Stellen Adenocarcinom. Riesenzellen, wenig Mitosen, beginnende Verhornung. Bindegewebe: hier und da Sklerose und hyaline Degeneration der Fibrillen, besonders in der Imgebung der Gefässe. Spärliche und wenig gut erhaltene elastische Fasern, in der Umgebung der Gefässe reichlicher. Kleinzellige Infiltration ungleichmässig, stellenweise stark und fast rein leukozytär.

7. III. 13. Nr. 3058. An der Oberfläche eine nekrotische Zone. Einzeln und in kleinen Häufchen liegende und zugrunde gehende Carcinomzellen mit gekörntem Protoplasma und blassen Kernen. Bindegewebe zeigt in ausgedehntem Masse eine hyaline Degeneration der Fibrillen. Reste glatter Muskelfasern. Wenig gut erhaltene, meist gequollene und blass gefärbte elastische Fasern. Kleinzellige Infiltration geringfügig und nur an der Oberfäsche. Intima der Gefässe verdickt, Adventitia sklerosiert.

11.III. 13. Nr.3069/70. Oberflächliche Nekrose. KeineCarcinomzellen mehr. Starke hyaline Degeneration der Fibrillen, die stellenweise breite homogene Massen unter Verlust aller Kerne bilden. Gefässe wie oben.

21. IV. Nr. 3142. Sehr viel nekrotisches Gewebe, in dem erhaltenen kein Carcinom. Granulationsgewebe. Hyaline Degeneration der Fibrillen. 
Aus dem Sektionsprotokoll (S. 413) sei das hier speziell Interessierende hervorgehoben: der Douglas ist durch Verklebungen des Rektums mit der hinteren Uteruswand verödet, die Flexur durch einige Adhäsionen an der linken Fundusecke fixiert. Vorn besteht eine etwa $1 \mathrm{~cm}$ breite, leicht zu lösende Verklebung des Blasenfundus mit der vorderen Uteruswand etwa $3 \mathrm{~cm}$ unterhalb des Fundus ateri, durch welche die weiter unten zu erwähnende Höhle gegen den freien Bauchraum abgeschlossen ist. Nach Herausnahme der Genitalien in Verbindung mit dem Rektum und der Blase zeigt sich folgendes: das untere Drittel der Vagina ist in seiner Form noch erhalten, die Schleimhautoberfläche grünlich verfärbt und schmutzig belegt. Aus ihr hängen gangränöse Fetzen heraus. An Stelle der oberen beiden Drittel der Vagina und der Cervix uteri findet man eine über faustgrosse mit schmieriggrünlichen Massen und gangränösen Gewebsfetzen gefüllte Höhle, die nach vorn zu durch die vordere Blasenwand (die hintere Blasenwand ist vollständig zugrunde gegangen) nach hinten durch das Rektum, nach den Seiten durch die stark infiltrierten Parametrien begrenzt wird. Die Vorderwand der Blase ist geschwollen, stark gerötet und stellenweise mit schmierig-gräulichen Massen belegt. Die Rektumschleimhaut zeigt eine trübe Schwellung. Die vordere Wand des Rektums ist an einer etwa $3 \mathrm{~cm}$ langen und $1,5 \mathrm{~cm}$ breiten Stelle, die etwa der Höhe der zerstörten Portio entspricht, papierdünn und steht vor der Perforation. In der linken Niere eine Carcinommetastase von etwa Kirschgrösse. - Die mikroskopische Untersuchung lässt folgenden Befund erheben: ein Schnitt durch den Uterus lässt in dem unteren der Höhle anliegenden Teile eine ausgedehnte Gewebsnekrose erkennen, welche die ganze Dicke der Wand umfasst. Allmählich nach dem Fundus zu und zuerst in den inneren Schichten, weiter oben auch in den mittleren und äusseren, macht das Gewebe wieder einen lebensfrischen Eindruck. Während in den unteren Abschnitten nur noch die sklerosierten Bindegewebsfibrillen besonders in der Adventitia gefärbt sind, alles andere ungefärbt geblieben ist, tritt in dem oberen Teile wieder eine Färbung aller Gewebebestandteile auf. Aber auch hier zeigen sich noch schwere Veränderungen; die Gefässe der sonst atrophischen Schleimhaut und der inneren Muskellagen sind stark gestaut, das Gewebe ödematös und mit Hämorrhagien durchsetzt, in denen es zum Teil zugrunde gegangen ist. Die innersten Schichten der Schleimhaut, die dem Lumen anliegen, sind nekrotisch, viele 
Gefässe thrombosiert und die Thromben teilweise vereitert. An einzelnen Stellen findet man noch Drüsen, deren Lumina mit Detritus angefüllt sind, dem hier und da noch als Zylinderepithel erkennbare Zellen beigemischt sind. Die Muskulatur zeigt ebenfalls schwere Veränderungen: sie ist neben wenigen gut erhaltenen Bündeln hyalin degeneriert, zum andern Teil aufgelöst in amorphe ungefärbte Massen von einem grau-grünlichen Ton (Eisenhämatoxylin (Weigert)-van Gieson-Färbung). Ebenso sieht die Media der Gefässe an vielen Stellen aus. Die Adventitia ist sklerosiert und hyalin degenerirt, die Intima verdickt. An vielen Gefässen finden sich ausgedehnte Verkalkungen der Media. Das Bindegewebe ist in weitgehendstem Umfang hyalin degeneriert mit fast völligem Verlust der Kerne. Irgendwelche deutlich erkennbare Reste von Carcinomgewebe finden sich nicht. In dem Septum recto-vaginale liegt die Rektummuskulatur nach der Scheide $z u$ frei und ist an einzelnen Stellen von Fetzen hyalin degenerierter zerfallender Bindegewebsfibrillen bedeckt. Von irgendwelchem Vaginalgewebe ist nichts mehr zu finden. Die Rektummuskulatur ist schwer geschädigt: die Kerne schmal und blass gefärbt, teilweise zugrunde gegangen, das Protoplasma unregelmässig gefärbt, bald blass, bald etwas dunkler, so dass ein ganz geflecktes Bild entsteht. An anderen Stellen ist die Schädigung noch weiter fortgeschritten. Die Kerne geschwunden, die Muskelfibrillen zerfallend, so dass vielfach nur noch amorphe Massen kaum gefärbten Protoplasmas vorhanden sind. Dazwischen finden sich Züge hyalin degenerierter Bindegewebsfibrillen, die ein lockeres Geflecht bilden, und zwischen denen keine Bindegewebskerne mehr nachweisbar sind. Die Rektumschleimhaut ist an der untersuchten Stelle schmal, die Drüsen schlecht erhalten, das Epithel zum grössten Teile abgestossen und zerfallend. Zwischen den Muskelzügen liegen in Hohlräumen einzelne kleine Nester von Zellen, deren Identität mit Carcinomzellen nur vermutungsweise anzunehmen ist. Die Zellen mit blassen Kernen und körnigem, zerfallendem Protoplasma liegen einzeln zwischen Detritusmassen zugrunde gegangener Zellen. Die Parametrien sind frei von Carcinom. Hingegen findet sich in der schon makroskopisch diagnostizierten Nierenmetastase lebensfrisches Carcinom.

Ueberblicken wir den Befund in diesem Falle, so können wir sagen, dass das Carcinom an dem Ausgangspunkte der Erkrankung zum Schwinden gebracht worden ist, denn es fanden sich nirgends 
mehr Nester von erhaltenem Carcinom. Aber dieses Zugrundegehen des Carcinoms wurde erkauft mit einer Nekrose aller übrigen Gewebebestandteile. Dort, wo sich noch Reste zerfallender Carcinomzellen fanden, im Septum rectovaginale, dort war das übrige Gewebe in etwa demselben Grade geschädigt. In die Nekrose war abgesehen von der primär ergriffenen Cervix und Vagina auch die ganze hintere Blasenwand einbezogen. Eine Fernwirkung auf die allerdings wohl erst in der letzten Zeit entstandene Metastase der Niere war nicht zu erkennen.

In dem zweiten zur Sektion gekommenen Fall handelte es sich um eine 37 jährige Frau V. mit einem Vaginalcarcinom. Die Patientin war von anderer Seite schon einmal operiert worden, doch war nicht zu erfahren, was gemacht worden war. Aus der Krankengeschichte sei hier folgendes entnommen: Aufnahme am 19. XI. 12. An der Vulva links findet sich ein gut verheilter Schuchardt-Schnitt. Die hintere Hälfte der Vagina ist durch ein kraterförmiges Geschwür eingenommen mit leicht blutender, aber ziemlich derber Wand; das Scheidenlumen wird soweit verengert, dass nur ein Finger knapp eindringen kann. Beiderseits ist das Parakolpium bis dicht an den Beckenknochen heran infiltriert, besonders breit und derb auf der rechten Seite. Uterus metritisch verdickt.

Die Patientin wurde nun zunächst 5 Tage lang mit Röntgenstrahlen behandelt, dann ausschliesslich mit Mesothorium, das mit einem dünnen Silberblech gefiltert wurde. Im ganzen erhielt die Frau per vaginam $185 \mathrm{H}$ und $28260 \mathrm{mgr}$-Std. Mesothorium. Klinisch trat insofern während der Behandlung eine Besserung ein, als der jauchige Fluor und die Blutungen aufhörten und einer rein schleimig-eitrigen Absonderung Platz machten, die fast ganz geruchlos war. Die Infiltrationen wurden weicher, der enge Scheidenkanal erweiterte sich, die Höhle nach rechts wurde grösser. Subjektiv fühlte sich die Patientin besser, da die Schmerzen Tage und Wochen ganz aussetzten. Es erfolgten aber einige Male schwere venöse Blutungen aus der rechten Höhle, welche die Patientin sehr herunter brachten. Nach einer sehr starken Blutung am 7. V., die sich in der Nacht vom 9.-10. V. wenn auch in leichterer Weise wiederholte, erfolgte der Tod unter den Zeichen der akuten Anämie.

Die Veränderungen während der Behandlung waren mikroskopisch kontrolliert worden: 
64 Haendly, Mit Mesothorium und Röntgenstrahlen behandelte Carcinome.

24. IX. Nr. 2936. Vor der Behandlung. Alveoläres Plattenepithelcarcinom. Beginnende Verhornung. Mässig reichlich Kernteilungsfiguren. Bindegewebe gut erhalten.

29. I. Nr. 3003. Carcinomzellnester ganz vereinzelt inmitten von sklerotischem Bindegewebe. Die Kerne der Carcinomzellen sind wechselnd gut tingiert. Protoplasma blass. Riesenzellen, einund mehrkernig.

11. III. Nr. 3067/68. Spärliche Nester von Carcinomzellen, Kerne verschieden gefärbt und von wechselnder Grösse. Protoplasma schlecht gefärbt: die Zellen befinden sich in den verschiedensten Stadien des Unterganges. Das Bindegewebe zeigt eine ausgedehnte Sklerose und hyaline Degeneration der Fibrillen mit Verlust der Bindegewebskerne. Kleinzellige Infiltration mässig.

19. IV. Nr. 3139. Granulationsgewebe, viel Nekrose. Keine Carcinomzellen.

Das uns aus dem Sektionsprotokoll (S. 415) Interessierende wäre folgendes: die Adnexe sind durch feste Adhäsionen im Douglas fixiert, der teilweise verklebt ist; rechtes Ovarium cystisch und mit der geschwollenen Tube verklebt, linkes Ovarium sehr derb. Bei der Herausnahme der Genitalien im Zusammenhang mit der Blase und dem Rektum wird rechts eine knapp faustgrosse Höhle eröffnet, deren laterale Wand zum Teil durch eine dem Beckenknochen aufliegende Schwiele, zum Teil durch den angerauhten Knochen selbst gebildet wird. Die Wand der Höhle ist teilweise graugrünlich verfärbt, teilweise mit gangränösen Gewebsfetzen belegt. In die Höhle ist die rechte Hälfte des oberen Teiles der Vagina und das rechte Parametrium aufgegangen. Der untere Teil der Vagina ist in seiner Form noch gut erhalten. Nach hinten zu wird die Höhle von der zum Teil sehr verdünnten Rektumwand gebildet. Von oben her mündet in die Höhle die Cervix; der Uterus ist auffallend blass und fühlt sich sehr derb an.

Aus den der Höhle anliegenden Geweben wurden an den verschiedensten Stellen Stücke zum Zwecke der mikroskopischen Untersuchung entnommen. Ueber das Resultat dieser Untersuchungen wäre zusammenfassend folgendes zu bemerken: an der der Höhle zuliegenden Fläche findet sich überall eine verschieden breite Zone, in der das Gewebe nekrotisch ist. Darunter liegt eine Schicht Granulationsgewebe, dazwischen hyalin degenerierte Bindegewebsfibrillen. Die Uterus- und Cervixmuskulatur weist eine schwere Degeneration auf: die Muskelbündel sind schmal, 
zum Teil hyalin degeneriert, zum andern Teil zeigen sie ein blass gefärbtes Protoplasma und atrophische helle Kerne. Das Bindegewebe ist sklerosiert und zeigt eine hyaline Degeneration der Fibrillen mit Schwund der Kerne. Die Gefässe weisen eine Sklerose und hyaline Degeneration der Adventitia und eine Schwellung der Intima und vielfach auch der Media auf. In dem Septum rectovaginale liegt die Rektummuskulatur nach der Scheide zu bloss, von Bestandteilen der Scheide ist nichts mehr zu entdecken. Die Rektummuskulatur ist zum Teil noch relativ gut erhalten, doch finden sich an vielen Stellen regressive Veränderungen. Die Rektumschleimhaut ist atrophisch und zeigt an'ganz wenigen Stellen das Drüsenepithel abgestossen im Lumen der Drüsen liegend. In der Mehrzahl der Drüsen finden sich überhaupt keine Epitheljen mehr. Das der Oberfläche naheliegende Gewebe ist unter der Nekrose stark kleinzellig infiltriert. Von Carcinomgewebe ist mit Sicherheit nur weniges mehr zu finden. Hier und da sieht man zwischen der Rektummuskulatur und -schleimhaut vereinzelt kleine Zellnester in Hohlräumen liegen, die teilweise mit Endothel ausgekleidet sind. Diese Zellen sind zum Teil verklumpt, das Protoplasma gekörnt, zerfallend, die Kerne klein, bald ganz dunkel, bald blass gefärbt, dazwischen Detritus. In der dem Beckenknochen aufliegenden Schwiele findet sich ein hyalin degeneriertes Bindegewebe ohne Kerne, dazwischen sehr viele Nervenquer- und längsschnitte und quergestreifte schlecht gefärbte Muskulatur. Von Carcinomgewebe findet sich hier nichts.

Im Uterus ist ein ausgedehnter Schwund und eine byaline Degeneration der Muskulatur neben einer Sklerosierung der Bindegewebsfibrillen zu konstatieren. Im Ovarium ist alles funktionierende Parenchym gesehwunden und durch hyalin degeneriertes Bindegewebe ersetzt.

Ueber diesen letzten unserer Fälle wäre zusammenfassend zu bemerken: Unter dem Einfluss der Mesothoriumbehandlung ist es zu einer Nekrose des carcinomatösen Gewebes gekommen, und zwar in einem so weitgehenden Masse, dass von den ursprünglichen Tumormassen nichts mehr zu finden ist. In dem restierenden Gewebe fanden sich keine lebensfrischen Carcinomzellen mehr, dagegen noch vereinzelt kleine Nester, in denen zwischen Detritus zugrundegehende Carcinomzellen lagen. Das diese Nester umgebende Gewebe aber wies in demselben Masse etwa, wie die Carcinomzellen selber, Zeichen des Unterganges auf. 
Alle die in der Literatur niedergelegten und diese von uns beobachteten Carcinomfälle haben das Gemeinsame, dass es bis auf zwei Ausnahmen zu einem ausgedehnten Untergang des Carcinomgewebes gekommen ist. Bei dem Untergang der Krebszellen finden sich die von allen Autoren einstimmig beschriebenen Veränderungen des Kerns und des Protoplasmas [Exner (7), Perthes (8), Kaiserling (9), Herxheimer und Reinke (10), Wickham und Degrais (4), Aschoff, Krönig und Gauss (2), Doederlein (3), Lossen (11), Verf. (5) etc. etc.]. Dieses Zugrundegehen der Careinomzellen fand aber nur unmittelbar unter dem bestrahlten Bezirke statt. Selbst die in nächster Nähe der bestrahlten Partie liegenden Carcinomzellen blieben zuweilen ebenso wie die Metastasen unbeeinflusst (Carcinom in den portalen LymphIrüsen, der Leber in dem Falle von Magencarcinom [Aschoff, Kroenig, Gauss (2)], Axillardrüsen in dem Falle von Mammacarcinom [dieselben], Nierenmetastase in unserem Falle (T. 413), von Cervixcarcinom). In den beiden Fällen von Carcinoma cervicis (Kroenig, T. 214), in denen sich noch ausgedehnte Massen von Carcinomgewebe gefunden haben, war eine Aenderung des Zellcharakters der Neubildung in dem Falle von Kroenig, und eine abnorme Sklerose und hyaline Degeneration des cervicalen Bindegewebes in der Umgebung des Carcinoms in unserem Falle festzustellen gewesen.

Am stärksten ist die Wirkung der Strahlen an der Oberfläche, wo es zumal bei der Anwendung des Mesothoriums zu oberflächlichen Nekrosen kommt. Während nun in unseren Fällen ein Ersatz des zugrundegegangenen Gewebes vereinzelt durch schwächliche Granulationen oder meistens durch sklerotisches, hyalin degeneriertes Bindegewebe erfolgt ist, haben wir in einem Falle (T. 462) eine ausgedehnte Neubildung von kräftigem zellreichem Bindegewebe gefunden.

Wie steht es nun aber mit der Tiefenwirkung? In dem Falle von Magencarcinom hat Kroenig (2) ohne Schädigung der Haut eine Vernichtung des Carcinoms in der bestrahlten Partie bis auf kleine Reste erreicht. Dagegen gelang es in keinem der anderen Fälle, ohne Schädigung der Oberfläche das Carcinomgewebe in der Tiefe zu beeinflussen. In dem Falle von Mammacarcinom (Kroenig) ist eine tiefe bis auf die Pleura reichende Nekrose zustande gekommen, trotzdem fanden sich noch einige Carcinomnester, die mehr der Oberfläche zu lagen. In dem 
Doederlein'schen Falle (3) von Cervixcarcinom hat sich in den obersten Schichten, in denen es zur Nekrose gekommen ist, kein Carcinom mehr gefunden; in der Tiefe aber, in den Parametrien, die zum mindesten der Beeinflussung durch Mesothoriumstrahlen hätten zugänglich sein müssen, hat sich auch nicht die geringste Veränderung an den Carcinomzellen erkennen lassen.

In den beiden von uns vor der Operation bestrahlten Fällen (T. 461, T. 462) ist es ebenfalls zu einer oberflächlichen Nekrose gekommen. Daneben sind aber deutliche Veränderungen nicht nur an den Carcinomzellen, sondern auch an den andern Geweben in der Tiefe der Uteruswand eingetreten. Die Wirkung ist jedoch keine gleichmässige. Es liegen neben untergegangenen und untergehenden Carcinomhaufen sowohl in der Tiefe wie auch unter der Oberfläche noch absolut lebensfrische Zellstränge (T. 461). Die verabreichte Strahlenmenge ist allerdings nicht sehr erheblich gewesen. Ein Einfluss auf die nächsto Etappe der Carcinommetastasierung, nämlich die hypogastrisehen Drüsen, fehlt. Bei der Anwendung der Röntgenstrahlen, die durch ein mehr oder weniger enges Bleiglasrohr einfallen, ist ja eine Beschränkung der bestrahlten Partieen nach den Seiten zu leider vorhanden. Bei dem hier aber ausschliesslich verwandten Mesothorium, das seine Strahlen nach allen Seiten aussendet, hätte man eine Beeinflussung erwarten sollen.

Am längsten und am ausgiebigsten sind die beiden zuletzt angeführten Carcinome, die Sektionsfälle (S. 413, S. 415), behandelt worden, und diese Präparate zeigen demgemäss die stärksten Veränderungen. Trotzdem haben wir bei diesen beiden Fällen in der Wand der Höhlen noch kleine Nester von Zellen gefunden, die als Carcinomzellen angesprochen werden müssen. Diese Zellen zeigen zwar kein lebensfrisches Aussehen mehr, sie gehen zugrunde, aber das Gewebe, in dem sie liegen, ist auch so schwer geschädigt, dass ein völliges Verschwinden des Carcinoms mit einem Untergange der übrigen Gewebselemente erkauft ist. Die Tiefenwirkung kann hier nicht mit Sicherheit beurteilt werden, denn es ist nicht möglich, zu entscheiden, wie weit die Strahlen oder die Infektion an der ausgedehnten Gangrän beteiligt sind. Bei der einen Patientin (S. 413) ist die Urininfiltration infolge der grossen Blasenfistel jedenfalls an dem umfangreichen Zugrundegehen des Gewebes mitbeteiligt. Auch bei der anderen Patientin (S. 415) hat vorübergehend eine Ureterfistel bestanden, die sich aber dann wieder spontan geschlossen hat. Bei beiden endlich war eine 
starke Verdünnung des Sept. rect. vag. vorhanden, die bei dem ersten Falle (S. 413) so weit ging, dass das Gewebe an einer zirkumskripten Stelle papierdünn vor der Perforation stand. Sehr interessant ist in dem zweiten Falle (S. 415) der Befund am Ovarium, auf den an anderer Stelle noch ausführlicher eingegangen werden soll. Es ist nämlich kein funktionsfähiges Parenchym mehr vorhanden, die Primärfollikel sind geschwunden und eine breite hyalin degenerierte Bindegewebsschicht überzieht die Oberfläche der Ovarien, auf der noch hier und da Epithelreste zu sehen sind. Das Keimepithel besitzt demnach anscheinend eine starke Affinität zu den Mesothoriumstrahlen und ist für die geringeren, in die Tiefe dringenden Mengen der Strahlen empfindlicher als die Carcinomzellen - es ist hier, wenn man so will, eine gute, ausgiebige Tiefenwirkung vorhanden.

Wir haben gesehen, dass die Tiefenwirkung bei der bisher gebräuchlichen Anwendung der Röntgen- und Mesothoriumstrahlen keine gleichmässige und keine genügende ist, um das Carcinom in der Tiefe der Uteruswand und der Parametrien sicher zu verniehten, ohne zugleich an der Oberfläche die schwersten Nekrosen zu machen. Es wird die Aufgabe der nächsten Zeit sein, festzustellen, ob durch starke Filtrierung ohne Schädigung der gesunden Gewebe eine erhöhte Wirkung der Strahlen in der Tiefe ermöglicht werden kann. Die klinischen Erfolge, welche wir mit diesem Verfahren in der letzten Zeit erzielt haben, sprechen dafür!

\section{Literatir.}

1. Bumm, Verh. d. Ges. f. Geb. u. Gyn. 1912. 25. Okt. - Berl, med. Ges. Sitzung v. 7.V. 13. - Dentsche Ges. f. Gyn. zu Halle. 1913. - Zentralbl. f. Gyn. 1912. Nr. 47.

2. Aschoff, Kroenig u. Gauss, Münch. med. Wochenschr. 1913. Nr. 7/8. - Deutsche Ges. f. Gyn. zu Halle. 1913.

3. Doederlein, Monatsschr. f. Geb. u. Gyn. 1912. Bd. XXXVII. H. 5. Deutsche Gesellsch. f. Gyn. zu Halle. 1913.

4. Wickham u. Degrais, Radiumtherapie. Deutsch von Max Winkler. Berlin. Springer. 1910. - Handbuch d. Radiumbiologie u. -therapie von P. Lazarus. Wiesbaden. Bergmann. 1913.

5. Haendly, Ges. f. Geb. u. Gyn. zu Berlin. 1912. Juni. - Berl. med. Ges. Sitzung v. 7. V. 13. - Deutsche Ges. f. Gyn. zu Halle. 1913.

6. Rob. Meyer, Ges. f. Geb. u. Gyn. 25. X. 12.

7. Exner, Wiener klin. Wochenschr. 1904. 
8. Perthes, Dentsche med. Wochenschr. 1904. Nr. 17/18.

9. Kaiserling, Handb. d. Radiumbiologie u. -therapie von Paul Lazarus. 1913.

10. Herxheimer u. Reinke, Erg. d. allg. Pathol. u. pathol. Anat. Lubarsch u. Ostertag. 1909. 13. Jahrg. II. Abt.

11. Lossen, Die biologischen Wirkangen der Röntgen- und Becquerelstrahlen. Urban u. Schwarzenberg. 1907.

\section{Erklärung der Abbildungen auf Tafeln IV-V.}

Figur 1. T. 461. 35jährige Frau H.W. Cervixcarcinom. 9350 mgr.-Std. Mesothorium. Medianer Sagittalschnitt durch einen Uterus mit Cervixcarcinom. Die rosa gefärbten Partien zeigen das nekrotische Gewebe, die rot gefärbten das erkennbare Carcinomgewebe an. Die in dem roten Felde belegenen feinen Striche und Punkte deuten die Stellen untergehender Carcinomzellen an, die dicker schwarz bezeichneten Partien das gut erhaltene Carcinom.

Figur 2. Stelle aus Figur 1, und zwar aus der Tiefe. Man sieht zwischen dem sklerotischen und hyalin degenerierten Bindegewebe Alveolen mit zerfallenden Carcinomzellen. Rechts unten noch ein Carcinomnest mit relativ gut erlaltenen Zellen.

Figur 3. Stelle aus Figur 1 dicht neben Figur 2. Man sieht in dem sklerosierenden Bindegewebe noch reichlich kleinere und grössere Carcinomalveolen mit meist gut erhaltenen Zellen liegen.

Figur 4. Metastase in einem Lymphknoten (Gl. hypogastrica) vom selben Fall. Rechts oben sieht man einen Teil des zerfallenden Zentrums der Metastase. Im übrigen ist das Carcinomgewebe gut erhalten. Links Reste des Lymphgewebes.

Figur 5. T. 462. 57jährige Fran Fr. Cervixcarcinom. 13320 mgr.-Std. Mesothorium. Schematischer Sagittalschnitt durch vordere Hälfte eines Uterus mit Korpusmyom und kraterförmigem, zerfallendem Cervixcarcinom. Die rot gehaltenen Stellen zeigen die Partien an, in denen sich noch Carcinomzellen, wenn auch meist zugrundegehende, mikroskopiseh fanden.

Figur 6. Stelle aus Figur 5. Kariolyse, völliger Zellzerfall, Leukozyteninfiltration.

Figur 7. Stelle aus Figur 5. Verhornung, syncytiale Umwandlung der Zellen, leukozytäre Infiltration.

Figur 8. Stelle ans Figur 5. Verhornung, Riesenzellen, Bindegewebsneubildung.

Figur 9. Stelle aus Figur 5. Starke hyaline Degeneration der Bindegewebsfibrillen, scirrhusähnliches Carcinom, syncytiale Umwandlung. 


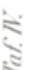

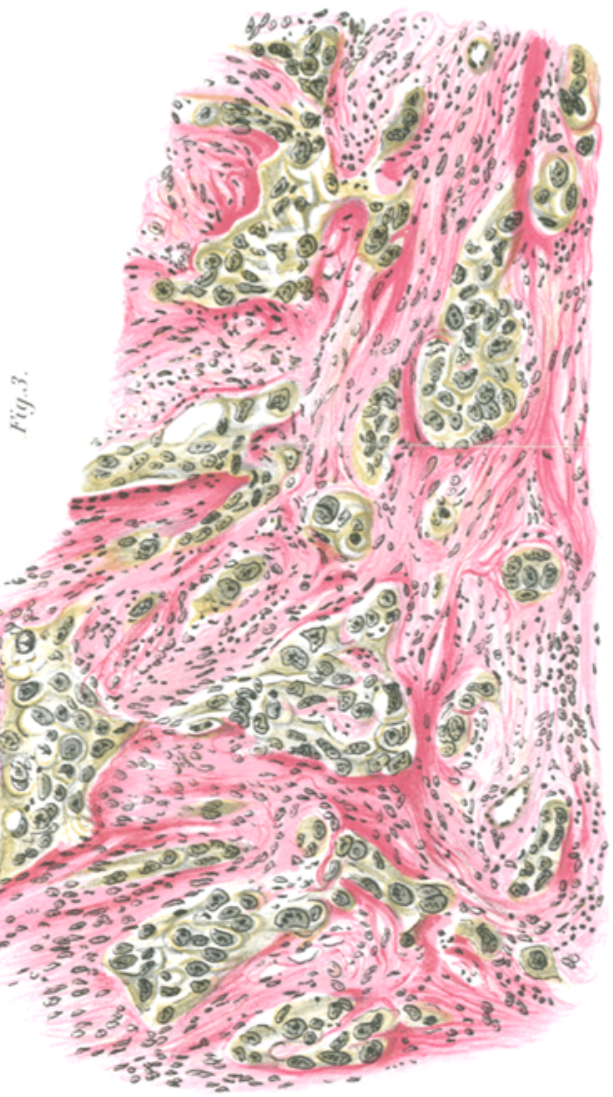

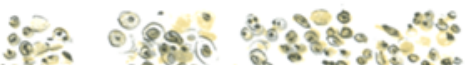
is of of

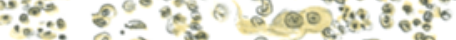

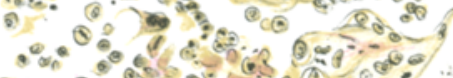

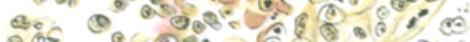

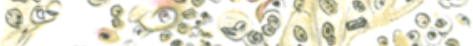

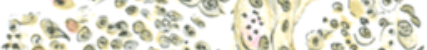

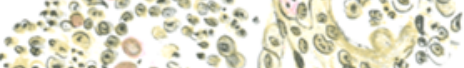
- of.

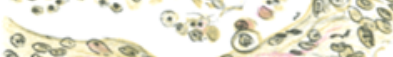

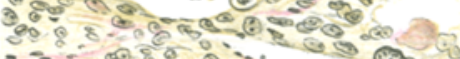

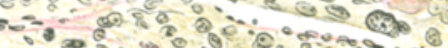

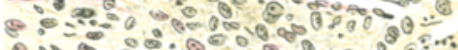

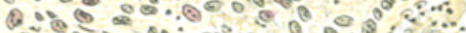
2.

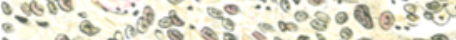

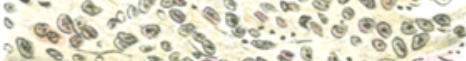

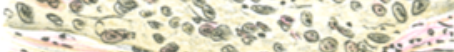

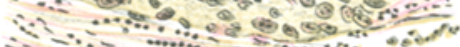

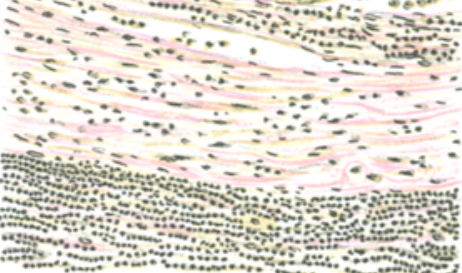

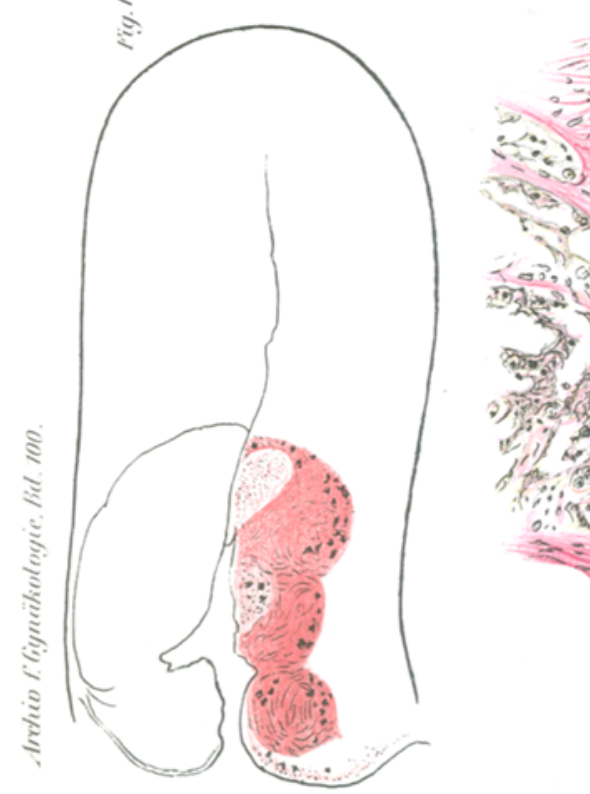

$0.97 .0 \%$

Wian:s,

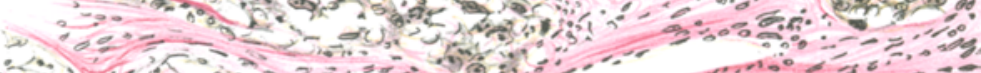

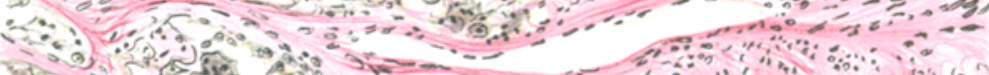

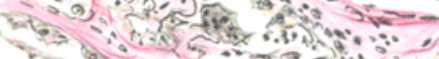

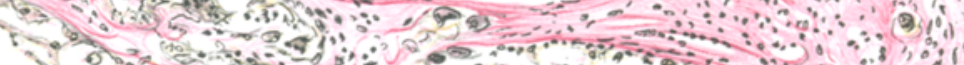

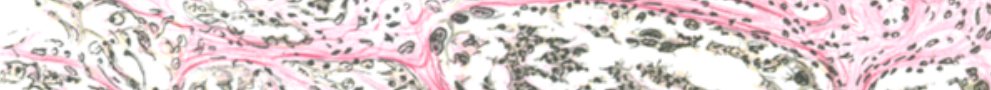

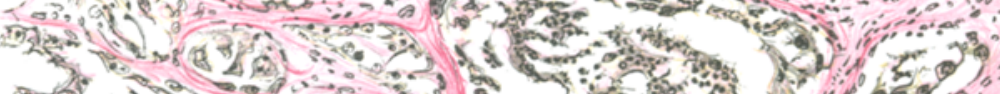

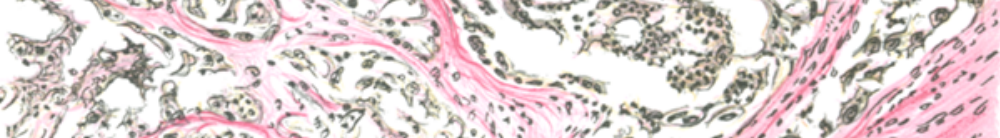

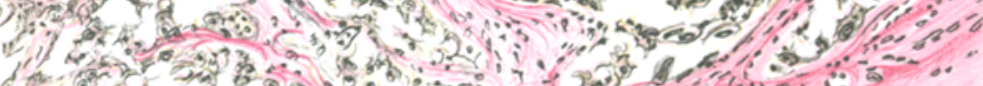

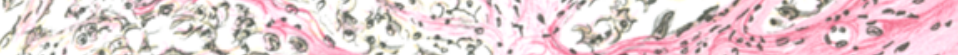

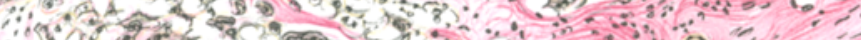
$\therefore$.

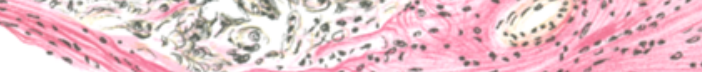

:

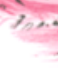



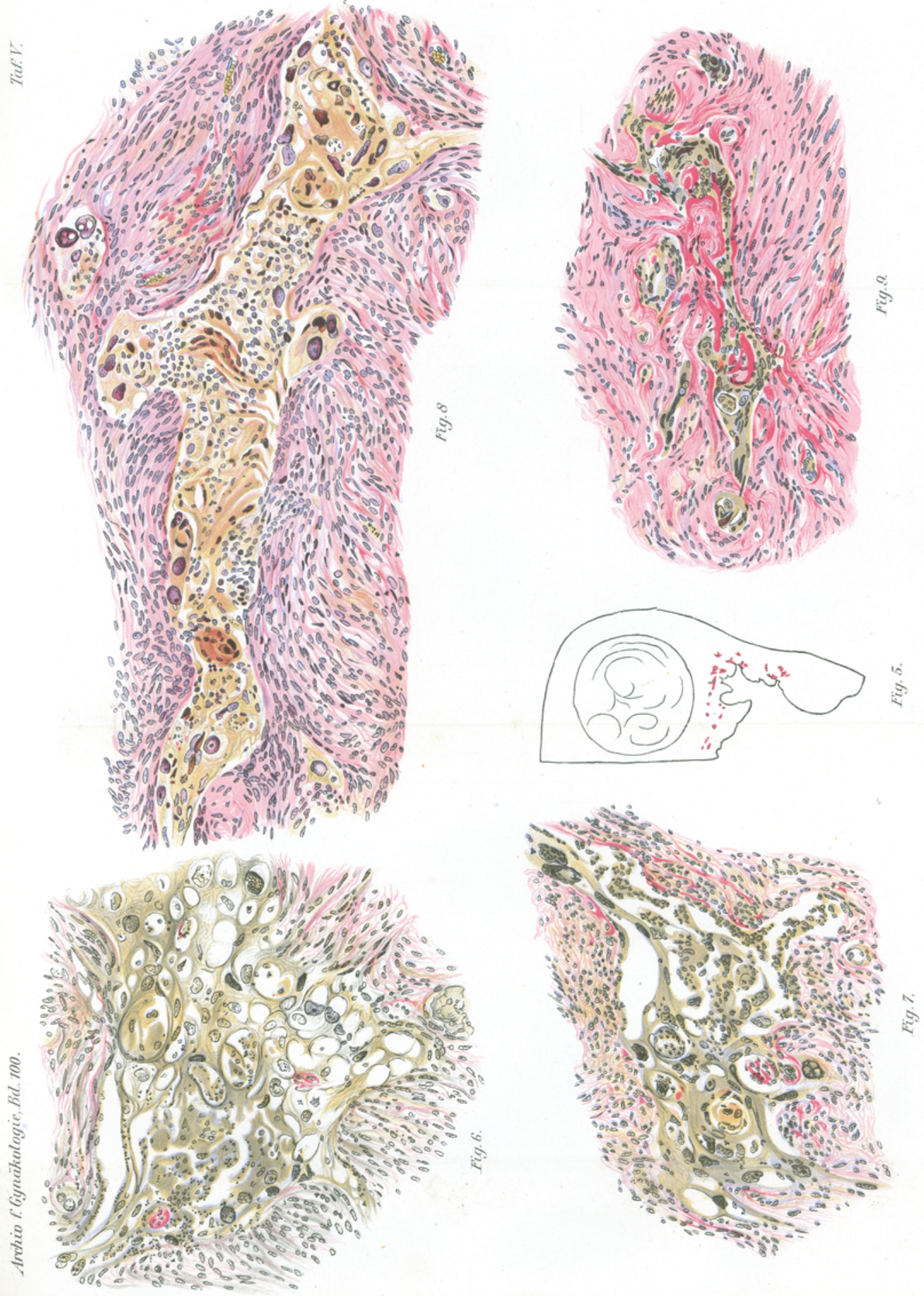